TITLE:

\title{
Use of probabilistic and deterministic measures to identify unfavorable earthquake records
}

\author{
$\operatorname{AUTHOR}(\mathrm{S}):$
}

Moustafa, Abbas; Takewaki, Izuru

\section{CITATION:}

Moustafa, Abbas ... [et al]. Use of probabilistic and deterministic measures to identify unfavorable earthquake records. Journal of Zhejiang University - Science A 2009, 10(5): 619-634

ISSUE DATE:

2009-05

URL:

http://hdl.handle.net/2433/89634

\section{RIGHT:}

c Springer. Springer Science+Business Media.; This is not the published version. Please cite only the published version.; この論文は出版社版で ありません。引用の際には出版社版をご確認ご利用ください。 


\title{
The use of probabilistic and deterministic measures to identify unfavorable earthquake records
}

\author{
Abbas Moustafa and Izuru Takewaki \\ Department of Urban \& Environmental Engineering, Graduate School of Engineering, Kyoto \\ University, Kyotodaigaku-Katsura, Nishikyo-ku 615-8540, Japan
}

\begin{abstract}
This paper develops measures to identify resonant or unfavorable earthquake ground motions. Probabilistic measures based on the entropy rate and the geometric properties of the power spectral density function of the ground acceleration are developed. Deterministic measures for the frequency content of the ground acceleration are also developed. The use of these measures to identify resonance in stochastic earthquake models and 110 acceleration records measured at rock, stiff, medium and soft soil sites is presented. The unfavorable earthquake record for a given structure is defined as the record having narrow frequency content and dominant frequency close to the structure fundamental natural frequency. Accordingly, the measures developed in this paper may provide a basis for selecting records that are capable of producing the highest structural response. Numerical verifications on damage caused to structures by identified resonant records are provided.
\end{abstract}

Keywords: entropy rate; dispersion index; power spectral density function; frequency content; unfavorable ground motion; resonant acceleration; critical accelerogram; energy rate; damage index. 


\section{INTRODUCTION}

Structural design to earthquake loads is the key tool for the mitigation of earthquake hazards. Structural engineers aim to design structures that are safe against possible future earthquakes and are economic at the same time. The specification of robust design earthquake loads for structures is the first step towards achieving this goal. The method of critical earthquake load modeling has been developed in the literature for specifying robust mathematical earthquake loads on structures. The studies by Abbas (2002, 2006) and Takewaki $(2002,2007)$ provide an extensive overview on this method. The critical or most unfavorable earthquake load for a given structure is derived by solving an inverse dynamic problem using optimization techniques subjected to predefined constraints reflecting known information on earthquake data at the site. These seismic loads are tailored to produce the highest structural responses while they satisfy predefined constraints on the earthquake ground motions at the site.

On the other hand, several studies have attempted to identify unfavorable real ground motion records (e.g. Anderson and Bertero 1987, Uang and Bertero 1988, Takewaki 2002, Amiri and Dana 2005, Dahakal et al 2006, Zhai and Xie 2007). Some of these methods, however, are conceptual, adopt sophisticated techniques or require nonlinear time history analysis, and, thus, are highly computational. For instance, Anderson and Bertero (1987) investigated the implications of the adjusted earthquake records on the maximum structural responses produced by near-field ground motions. Takewaki (2001) used the critical excitation method to quantify resonance and criticality of earthquake records for a given structure by comparing the structural response produced by the critical input and that from the earthquake record. Dana and Amiri (2005) proposed the effective peak ground velocity to identify resonant records at a given site. The study by Dhakal et al (2006) employed probabilistic methods to identify critical earthquake records and tried to relate them to the maximum design earthquake. The study by Zhai and Xie (2007) employs the critical excitation concept to identify unfavorable earthquake records for structures of known 
frequency range. The method developed by these authors, however, requires nonlinear time history analysis of the structure under each record, and is thus highly computational (Moustafa 2008).

Abbas and Manohar (2002, 2005, 2007) examined the significance of incorporating a lower bound on the entropy rate of the ground acceleration in deriving critical random earthquake load models. These studies showed the significance of the entropy rate constraint quantified from actual recorded accelerograms in producing realistic earthquake loads that are rich in frequency content.

In this paper, we employ the notion of the critical excitation method and random vibration theory to develop measures for identifying resonant or unfavorable earthquake records among a set of records. The first measure is based on the concept of the entropy of random processes and the second measure is the dispersion index of the power spectral density function (PSDF) of the ground acceleration and is based on the work of Vanmarcke $(1972,1976)$. Deterministic measures of the frequency content of the ground acceleration are also developed. These measures can provide a basis for the selection of proper design records for structures. Numerical illustrations on identification of resonance in random process and 110 earthquake records at various soil sites and different earthquake characteristics are provided.

The next section demonstrates the development of a new measure using the entropy rate for identifying resonance in random processes. Subsequently, the dispersion index and central frequency measures developed by Vanmarcke are explained. The use of these measures to identify resonant earthquake records is then demonstrated. The developments of deterministic measures for identifying frequency content of ground motions is then explained. The last section illustrates the use of these measures for the selection of proper acceleration records for seismic design of structures.

ENTROPY AS A MEASURE OF RESONANCE AND CRITICALITY OF PROBABILISTIC EARTHQUAKE MODELS

The idea of using entropy to measure the amount of information in random signals sent 
along a transmission line was proposed by Shannon (1948). This idea has been advocated as being a general principle of statistical inference and has been used in science, engineering and economics. The literature on the use of entropy in engineering is vast (see, e.g., Papoulis 1991, Kapur 1993). Entropy in its basic form is a measure of uncertainty or missing information. For instance, the entropy of a random variable $x$ is a measure of the uncertainty associated with that random variable, which is given in terms of the probability density function $p(x)$ as follows (Papoulis 1991):

$$
H_{x}=\int_{-\infty}^{\infty} p(x) \ln p(x) d x
$$

When $H_{x}$ is large, the uncertainty as to the value of $x$ is also large. For instance, the entropy of a normal random variable of large coefficient of variation is also large. Note that when the coefficient of variation is significantly large, the normal distribution can approximate the uniform distribution that possesses the largest entropy among all distributions. On the other hand, when the coefficient of variation is very small, the distribution may approximate a deterministic quantity which possesses zero entropy.

In the context of earthquake engineering, the use of entropy was introduced in modeling critical earthquake loads (Manohar and Sarkar 1995, Abbas 2002, Abbas and Manohar 2002, 2007). These studies proved the crucial role of including the entropy in producing realistic critical earthquake loads. The entropy of a zero-mean stationary Gaussian random process $\ddot{u}(t)$ is given as (Papoulis 1991):

$$
H=\ln \sqrt{2 \pi e}+\frac{1}{2\left(\omega_{u}-\omega_{0}\right)} \int_{\omega_{0}}^{\omega_{u}} \ln S(\omega) d \omega
$$

where $\left(\omega_{0}, \omega_{u}\right)$ and $S(\omega)$ define the frequency range and the one-sided PSDF of the ground acceleration. The constant $\ln \sqrt{2 \pi e}$ represents a reference level from which entropy rate is measured. Eq. (2) reveals that the entropy rate of a stationary Gaussian process is constant. Thus, when the entropy rate of $\ddot{u}(t)$ is large the uncertainty as to the value of $\ddot{u}(t)$ 
at discrete time instants is also large. On the other hand, the entropy of a harmonic signal with a random amplitude and a single or very few frequencies (e.g., $\left.\ddot{u}(t)=A \sin \left(\omega_{g} t\right)\right)$ is almost zero. In other words, the energy of the signal or the amplitude of the Fourier transform of a sine or a cosine time series of a single or very few frequencies is well ordered in the frequency range $\left(\omega_{0}, \omega_{u}\right)$ while a time signal composed of several frequencies will be disordered. Note that two random processes with the same energy (i.e., same area under the PSDF) need not possess the same entropy. This is because entropy depends on the frequency bandwidth and the spectral amplitude of the PSDF (see Eq. 2). To gain more insights into the use of entropy in characterizing ground motions we derive the entropy of probabilistic models of Gaussian ground motion models in the next subsections.

\section{Stationary narrow-band white noise model}

The narrow-band random process has been used extensively as an idealization for random signals, noises, turbulences and earthquakes (Lin 1967, Nigam and Narayanan 1994). Consider a stationary narrow-band signal of intensity $s_{0}$ and central frequency $\omega_{c}$. Thus, Eq. (2) leads to:

$$
H=\frac{\ln s_{0}}{2\left(\omega_{u}-\omega_{0}\right)}
$$

Therefore, the parameters $s_{0}, \omega_{0}, \omega_{u}$ define the entropy of the narrow-band signal. Note that the term $\ln \sqrt{2 \pi e}$ was omitted in Eq. (2).

\section{Stationary band-limited white noise model}

The band-limited white process has been used as an approximation for earthquake and wind loads. This process possesses finite energy, constant spectral amplitude in the frequency range $\left(\omega_{0}, \omega_{u}\right)$. Thus, Eq. (2), leads to:

$$
H=\frac{\ln s_{0}}{2}
$$

Accordingly, the spectral parameter $s_{0}$ defines the entropy of the band-limited process. 
Equations (3) and (4) reveal that entropy of the band-limited ground acceleration model is significantly larger than that of the narrow-band acceleration model.

\section{Stationary Kanai-Tajimi model}

This model has been widely used in modeling strong ground motions. The PSDF of the ground acceleration is obtained by passing a band-limited white noise through a filter that represents the soil layer above the bed-rock, given as (Kanai 1957, Tajimi 1960):

$$
S(\omega)=s_{0} \frac{\omega_{g}^{4}+4 \eta_{g}^{2} \omega_{g}^{2} \omega^{2}}{\left(\omega_{g}^{2}-\omega^{2}\right)^{2}+4 \eta_{g}^{2} \omega_{g}^{2} \omega^{2}}
$$

where, $s_{0}, \eta_{\mathrm{g}}, \omega_{\mathrm{g}}$ are the intensity of the PSDF at the rock level, damping and frequency of the soil layer, respectively. The entropy of the Kanai-Tajimi model can be estimated numerically instead of integrating Eq. (2) analytically.

\section{Nonstationary and evolutionary PSDF models}

Several earthquake acceleration models have been developed in the literature to account for nonstationarity in time and frequency content. This class of earthquake models is known as evolutionary PSDF models (see, e.g., Nigam and Narayanan 1994 for more details). Herein, the PSDF of the ground acceleration is a function of time and frequency and is represented as:

$$
S(t, \omega)=|A(t, \omega)|^{2} S(\omega)
$$

where $A(t, \omega)$ is a modulating envelope that could be a complex function and $S(\omega)$ is a stationary PSDF. When $A(t, \omega)$ is separable into a time function and a frequency function, the model reduces to the uniformly modulated nonstationary random process which possesses invariable PSDF at all time instants. Accordingly, entropy is constant and the computation follows the same procedures for stationary acceleration models. Consider the ground acceleration defined by the Kanai-Tajimi PSDF of Eqs. (5) and (6) with $A(t, \omega)$ given as:

$$
A(t, \omega)=e(t) g(t, \omega)=A_{0}\left[e^{-\alpha t}-e^{-\beta t}\right] e^{\left(\frac{-r \omega t}{\omega_{m} t_{m}}\right)}
$$

where, $A_{0}, \alpha, \beta, r, \omega_{m}, t_{m}$ are constants. Herein, the quantification of entropy can be carried 
out using numerical integration of the evolutionary PSDF at discrete points of time. Fig. 1 shows the evolutionary PSDF of the ground acceleration and the associated entropy function for $A_{0}=2.87, \alpha=0.13, \beta=0.35, r=1.0, \omega_{m}=5.0 \mathrm{~Hz}$ and $t_{m}=5.0 \mathrm{~s}$.

The above subsections demonstrated the quantification of the entropy for probabilistic earthquake models. It is shown that the entropy for stationary and uniformly modulated random processes is constant. Also, the entropy of the band-limited acceleration is significantly larger than that of the narrow-band acceleration. We explain the quantification of the relative entropy of two random processes in the next subsection.

\section{Relative entropy of two random processes}

To compare entropy from alternative acceleration models, we measure entropy of the random process $\ddot{u}(t)$ with reference to a wide-band signal $\ddot{z}(t)$ of spectral intensity $s_{0}$. This is known as the relative entropy of two random processes. Thus, under the assumption that $\ddot{u}(t)$ is independent of $\ddot{z}(t)$, the increase in entropy when $\ddot{u}(t)$ is added to $\ddot{z}(t)$ is given as:

$$
\Delta H=\frac{1}{2\left(\omega_{u}-\omega_{0}\right)} \int_{\omega_{0}}^{\omega_{u}} \ln \left[1+\frac{S(\omega)}{s_{0}}\right] d \omega
$$

We now calculate the entropy index $\Delta H$ for the narrow-band, the Kanai-Tajimi and the band-limited acceleration models, described above, from a reference wide-band signal of intensity $0.02 \mathrm{~m}^{2} / \mathrm{s}^{3}$. The PSDF for each of these three models is normalized such that they possess unit area (see Fig. 2). This normalization implies equality of the earthquake energy of the three models (Arias 1970). The parameters of the Kanai-Tajimi model $\omega_{g}, \eta_{g}$ are taken as $\pi \mathrm{rad} / \mathrm{s}, 0.20$ for soft soil, $3 \pi \mathrm{rad} / \mathrm{s}, 0.40$ for medium soil, $6 \pi \mathrm{rad} / \mathrm{s}, 0.60$ for stiff soil, and $9 \pi \mathrm{rad} / \mathrm{s}, 0.80$ for rock soil (see, Table 1). Additionally, the spectral intensity at the rock level is taken as $s_{0}=0.02 \mathrm{~m}^{2} / \mathrm{s}^{3}$ and the central frequency of the narrow-band signal is taken as $\omega_{g}=\pi, 3 \pi, 6 \pi, 9 \pi \mathrm{rad} / \mathrm{s}$ for soft, medium, stiff, and rock soil, respectively. The numerical results are shown in Table 1. Based on careful examination of these results, the 
following observations are made:

(1) The narrow-band acceleration possesses the smallest entropy. In other words, the PSDF of this model is well ordered or the acceleration energy is concentrated at a single frequency. Note that the central frequency of the acceleration does not influence the value of the entropy (see Table 1 and Eq. 3). Thus, entropy of narrow-band signals with the same energy is invariant regardless of the central frequency.

(2) The band-limited acceleration possesses the highest entropy among all models. This is because the energy of the process is well represented at all frequencies.

(3) The Kanai-Tajimi acceleration is significantly disordered. This is expected since the PSDF is reasonably distributed across a significant frequency range (see Fig. 2a). The entropy for soft soil is small compared with that for rock soil. This is not surprising since the PSDF for soft soil is narrow-band while that for rock soil is distributed across a wider frequency range (Fig. 2b).

(4) Entropy of the Kanai-Tajimi model is bounded between entropy of the narrow-band acceleration and that from the band-limited acceleration for all soil conditions (see Table 1). This result is interesting since it provides lower and upper bounds on entropy of the Kanai-Tajimi acceleration model.

In general, earthquake ground motions possess amplitude distributed across a significant frequency range. This is because the energy released at the source gets amplified and filtered by the soil layer above the rock level due to site and attenuation effects caused by soil damping, geometric spreading, wave scattering and local soil profile. As discussed in the Introduction section, real accelerograms, however, exhibit the resonance trend, and thus, the associated frequency range is narrow which can be characterized in terms of the entropy.

This section explained the use of entropy as a measure of the frequency content of probabilistic earthquake models. It is shown that the narrow-band and the band-limited signals provide lower and upper bounds on the entropy of the Kanai-Tajimi model. We estimate entropy of recorded accelerograms in the next section. 


\section{DISPERSION INDEX AND CENTRAL FREQUENCY}

Vanmarcke $(1972,1976)$ developed measures for the frequency content of the ground acceleration in terms of the geometric properties or the moments of the PSDF. These measures are outlined here. The $i$ th moment of $S(\omega)$ is given as:

$$
\lambda_{i}=2 \int_{0}^{\infty} \omega^{i} S(\omega) d \omega ; i=0,1, \ldots . n
$$

The zeroth moment defines the energy and the second moment defines the variance of the random process. The $i$ th frequency is defined as:

$$
\omega_{i}=\left(\frac{\lambda_{i}}{\lambda_{0}}\right)^{\frac{1}{i}} ; i=1,2, \ldots ., n
$$

Here $\omega_{1}=\omega_{c}$ is the central frequency of the ground acceleration and $\omega_{2}$ indicates where the spectral mass of the PSDF is located along the frequency range. The radius of gyration of $S(\omega)$ about the frequency origin $\omega_{c}$ is given as (Vanmarcke 1972,1976):

$$
\omega_{s}=\sqrt{\omega_{2}^{2}-\omega_{1}^{2}}
$$

$\omega_{s}$ is a measure of dispersion of the PSDF about the central frequency. Thus, when $\omega_{s}$ is small it implies that the ground acceleration is narrow-band and when $\omega_{s}$ is large, the ground acceleration is broad-band or rich in frequency content. Table 1 summarizes the dispersion index for narrow-band, Kanai-Tajimi, and band-limited random processes defined earlier. The numerical values of the parameters of these models are given in the same table. It is evident from these results that the narrow-band and the band-limited models provide lower and upper bounds on the dispersion index of the Kanai-Tajimi model. The next section demonstrates the use of the measures developed in this section and the previous section for identifying resonant earthquake records. 


\section{EARTHQUAKE RECORDS}

Consider an actual recorded earthquake acceleration $\ddot{x}(t)$ that is represented as:

$$
\ddot{x}(t)=e(t) \ddot{u}(t)=A_{0}\left[e^{-\alpha t}-e^{-\beta t}\right] \ddot{u}(t)
$$

Here $\ddot{u}(t)$ represents a steady-state function and $e(t)$ is an envelope function that defines the nonstationarity of $\ddot{x}(t)$. The envelope parameters $A_{0}, \alpha_{1}$ and $\alpha_{2}$ can be estimated by matching the transient trend of the earthquake acceleration. Subsequently, the stationary part $\ddot{u}(t)$ can be obtained by dividing $\ddot{x}(t)$ by $e(t)$. The PSDF of $\ddot{u}(t)$ can then be calculated. This is followed by the estimation of the entropy using Eq. (8).

Fig. (4) shows the PSDFs for four ground acceleration models. The first acceleration represents a sample narrow-band signal $\ddot{x}(t)=e(t) A \sin \omega_{g} t$, with random amplitude $A$ and central frequency $\omega_{g}=3 \pi$ (medium soil). The second acceleration represents a sample of a band-limited model, $\ddot{x}(t)=e(t) \sum_{i=1}^{N} A_{i} \cos \left(\omega_{i} t\right)+B_{i} \sin \left(\omega_{i} t\right)$, where $\quad A_{i} \quad$ and $\quad B_{i}$ are uncorrelated normal random variables of variance $s_{0}$. The third signal is a simulated acceleration from the Kanai-Tajimi model of Eq. (5) for medium soil $\left(\omega_{g}=3 \pi \mathrm{rad} / \mathrm{s} ; \eta_{g}=0.40\right)$. The fourth acceleration represents the first horizontal acceleration of the 1992 Cape Mendocino (Petrolia) earthquake measured at medium soil site (PEER 2005). The stationary components $\ddot{u}(t)$ for the first three models are modulated by the envelope function that matches the transient trend of the actual record and all accelerations are normalized to unit intensity.

The relative entropy of these accelerations from a wide-band acceleration of spectral intensity $0.02 \mathrm{~m}^{2} / \mathrm{s}^{3}$ are determined. The numerical values were found to be 0.03 for the narrow-band acceleration, 0.56 for the band-limited acceleration, 0.19 for the simulated Kanai-Tajimi acceleration and 0.32 for the Cape Mendocino accelerogram (see Table 1). These results reveal that entropy of the Kanai-Tajimi model is bounded by entropy of the narrow-band acceleration and entropy of the band-limited acceleration. Interestingly, entropy of the recorded acceleration is also bounded by the narrow-band and the band-limited signals. Note that the narrow-band signal represents a resonant acceleration that is poor in frequency 
content. The band-limited signal, on the other hand, represents an acceleration that is rich in frequency content. Based on this observation it can be expected that entropy of a resonant or a narrow-band acceleration will be the smallest among a set of records while, entropy of an acceleration that is rich in frequency content will be large. The next section develops deterministic measures to quantify the frequency content of ground motions.

THE USE OF DETERMINISTIC MEASURES TO IDENTIFY RESONANCE OF EARTHQUAKE RECORDS

Consider an acceleration record of finite energy in time domain satisfying the condition:

$$
E_{t}=\int_{-\infty}^{\infty}[\ddot{x}(t)]^{2} d t<\infty
$$

Under this condition, the Fourier transform of the ground acceleration is given by:

$$
y(\omega)=\int_{-\infty}^{\infty} \ddot{x}(t) e^{-i \omega t} d t
$$

Equation (13) provides a measure of the acceleration energy computed in time domain (Arias 1970). Recalling Parseval's theorem $\left(\int_{-\infty}^{\infty}[\ddot{x}(t)]^{2} d t=\frac{1}{2 \pi} \int_{-\infty}^{\infty}|y(\omega)|^{2} d \omega\right)$, a similar measure can be computed in frequency domain:

$$
E_{\omega}=\int_{-\infty}^{\infty}|y(\omega)|^{2} d \omega=\int_{-\infty}^{\infty} y(\omega) y^{*}(-\omega) d \omega
$$

Herein, $y^{*}(-\omega)$ is the complex conjugate of $y(\omega)$. The frequency content, proposed in this paper, is taken as $\left(\omega_{a}, \omega_{b}\right)$ where $\omega_{a}$ and $\omega_{b}$ represent the frequencies at which $a$ and $b$ times the Fourier energy are attained, respectively. Thus, the frequency-bandwidth is taken to be given as $\omega_{e f}=\omega_{b}-\omega_{a}$ (see Fig. 4). Typical values of $a$ and $b$ can be taken as 0.05 and 0.95 (5\% and $95 \%$ of the acceleration energy), respectively or any reasonable values (e.g., 0.01 and 0.99). When $a=0.05$ and $b=0.95, \omega_{e f}$ can be viewed as a measure of the frequencies contributing to the strong phase of the ground motion (see, e.g. Trifunac and 
Brady 1975). The effective frequency-bandwidth can be further normalized to provide a measure that is bounded between zero and one:

$$
\bar{\omega}_{e f}=\frac{\omega_{b}-\omega_{a}}{\Omega_{u}-\Omega_{0}}
$$

The frequency range $\left(\Omega_{0}, \Omega_{u}\right)$ is generally in the range of $2 \pi(0,10 \sim 50) \mathrm{rad} / \mathrm{s}$ depending on the site soil condition. When $\bar{\omega}_{e f}$ is close to zero, the ground acceleration is narrow-band or poor in frequency content. An example of this scenario is a harmonic signal of a single or a few frequencies (e.g., $\ddot{x}(t)=A \sin \left(\omega_{g} t\right)$, where $A$ and $\omega_{g}$ are the acceleration amplitude and dominant frequency, respectively). When $\bar{\omega}_{e f}$ is significantly larger than zero, the ground acceleration will be rich in frequencies. The band-limited acceleration model (constant amplitude at all frequencies) is an example of this scenario. The average frequency of the ground motion is calculated as $\omega_{a v}=\left(\omega_{b}-\omega_{a}\right) / 2$.

The central frequency or dominant frequency of the ground acceleration that reflects the influence of the local soil profile can be computed as:

$$
\omega_{c}=\int_{-\infty}^{\infty} \omega|y(\omega)|^{2} d \omega / \int_{-\infty}^{\infty}|y(\omega)|^{2} d \omega
$$

An additional frequency $\omega_{\max }$ that corresponds to the peak amplitude of $y(\omega)$ can be also estimated (see Fig. 4). The relevance of $\omega_{\max }$ becomes obvious by comparing $\omega_{\max }$ with the fundamental frequency of the structure. For instance, when the ratio $\omega_{\max } / \omega_{n}$ is close to one, it can be expected that the input energy to the structure will be large (Takewaki 2004).

It is believed that these measurers provide important information on the nature of the ground motion, and, thus, can be adopted in identifying the frequency content of recorded accelerograms. It is proposed in this paper that these measures be utilized in selecting recorded accelerations as design inputs to important structures. Thus, if a set of $n$ records are available and it is required to select a few records (typically 3 10) for seismic design of a new 
structure of fundamental frequency $\omega_{n}$ (obtainable using approximate or empirical expressions), the following procedures can be adopted:

(1) Normalize the set of available accelerograms to the same Arias intensity (Arias 1970).

(2) Calculate the central and effective frequencies for each record (Eqs. 16, 17).

(3) Sort the records based on the central frequency and associated effective frequency.

(4) Select those records that have their $\omega_{c}$ close to $\omega_{n}$ and have the smallest $\bar{\omega}_{e f}$.

To demonstrate the use of the measures developed in this paper in quantifying resonant recorded accelerograms, we consider the acceleration records listed in Table 2 (PEER 2005). The numerical values of these measures are given in the same table. These results reveal the significant differences of these records. It is seen that $\bar{\omega}_{e f}$ for the Chichi record is the smallest and that for the Landers is the largest. The Kobe record would govern the design of buildings of $\omega_{n}$ in the range $(0,4) \mathrm{Hz}$. This observation is confirmed by the large Fourier amplitude of the Kobe record that is about twice the amplitude of El Centro record and about four times that of the Chichi record.

\section{IDENTIFICATION OF RESONANT ACCELERATIONS AND SELECTION OF DESIGN} ACCELEROGRAMS

To examine the applicability of the measures developed in this study in identifying resonant accelerograms at a site we consider the four recorded earthquake groups shown in Table 3. These records include accelerograms measured at rock, stiff, medium and soft soil sites (PEER 2005). The selection of these records is based on the site soil classification adopted by the USGS in terms of the shear wave velocity $v_{\mathrm{s}}$ (see Table 3 ). The vertical and the two horizontal accelerations of each earthquake are considered in the numerical analyses. Table 3 provides information on magnitude, source-site distance, PGA, Arias intensity, duration and recording station for the 72 accelerograms considered. In numerical calculations, the dispersion index is normalized by $\omega_{2}$ and all records are scaled to the same Arias intensity.

The numerical results on entropy and dispersion indices for these records are provided in 
Table 3. The mean and coefficient of variation for the entropy are $(0.58,0.17),(0.52,0.23)$, $(0.47,0.14)$, and $(0.50,0.28)$ for rock, stiff, medium and soft soil, respectively. The higher variation is seen in the entropy for the soft soil site which could be attributed to the differences in the source properties and other characteristics (e.g., duration, magnitude, epicentral distance, fault mechanism, etc.). If the 72 accelerograms are considered, the mean entropy and coefficient of variation are calculated as $(0.52,0.22)$. Figs. 5-8 depict the PSDF for the stationary components of the vertical and the two horizontal accelerations, respectively. The results reveal that the entropy and the dispersion indices correlate well and that they successfully identify resonant accelerograms. For instance, the vertical acceleration of the 1979 Imperial Valley earthquake recorded at soft soil (El Centro \#3) possesses the lowest entropy among all records. Fig. 5 confirms this result since the PSDF of this acceleration is narrow-band and resembles a resonant signal with high amplitude at a single frequency. On the other hand, the accelerations that are rich in frequency content (e.g. 1999 Kocaeli (Arcelik) earthquake) possess the largest entropy. It is also evident that the three accelerations of the same earthquake have different entropy and dispersion indices. The Fourier transform of these accelerations were seen to confirm these observations.

The numerical results reveal also that the Arias intensity and the peak ground acceleration are not guaranteed as accurate parameters for selecting design ground motions for structures. For instance, the vertical acceleration of the 1992 Landers earthquake (Lurcene) measured at rock soil possesses high PGA $(0.82 \mathrm{~g})$ and high Arias intensity $\left(51.37 \mathrm{~m}^{2} / \mathrm{s}^{3}\right)$. However, the spectral amplitude of this acceleration is substantially small in the frequency range $2 \pi(0,9)$ $\mathrm{rad} / \mathrm{s}$. On the other hand, the vertical acceleration of the 1992 Cape Mendocino (CM) measured at the same soil condition has relatively lower PGA $(0.75 \mathrm{~g})$ and substantially smaller intensity $\left(8.59 \mathrm{~m}^{2} / \mathrm{s}^{3}\right)$ but possesses significantly higher spectral amplitudes in the frequency range $2 \pi(0,2) \mathrm{rad} / \mathrm{s}$. Thus, it can be expected that this record will produce large deformations in a structure with short to moderate period compared to the first acceleration. We verify this observation below. 
Consider three SDOF buildings of natural frequency of $0.13,0.21$, and $0.41 \mathrm{~Hz}$. The yield displacement and yield strength are taken as $u_{y}=0.10 \mathrm{~m}$ and $f_{y}=10^{4} \mathrm{~N}$ for the three structures and a viscous damping of 0.05 damping ratio is adopted. The material nonlinearity is modeled using elastic-plastic stress-strain relation. Nonlinear dynamic analysis is carried out for each structure subjected to a single acceleration using the Newmark $\beta$-method $(\alpha=1 / 2$, $\beta=1 / 6, \triangle t=0.004)$. All records were normalized to the same Arias intensity of $6.00 \mathrm{~m}^{2} / \mathrm{s}^{3}$. The Park and Ang damage index for each structure driven by the ground acceleration is calculated as follows (Park et al., 1985):

$$
D I_{\mathrm{PA}}=\frac{u_{\mathrm{max}}}{u_{\mathrm{u}}}+\beta \frac{E_{\mathrm{H}}}{f_{\mathrm{y}} u_{\mathrm{u}}}=\frac{\mu_{\mathrm{max}}}{\mu_{\mathrm{u}}}+\beta \frac{E_{\mathrm{H}}}{f_{\mathrm{y}} u_{\mathrm{y}} \mu_{\mathrm{u}}}
$$

Here $u_{\max }$ and $E_{\mathrm{H}}$ are the maximum displacement and dissipated hysteretic energy (excluding elastic energy) under the earthquake (Abbas 2006). $\mu_{\mathrm{u}}$ is the ultimate yield ductility capacity under monotonic loading and $\beta$ is a positive constant that weights the effect of cyclic loading on structural damage (taken as 6 and 0.15). The numerical results on $D I_{\mathrm{PA}}$ are provided in Table 4. From the numerical results it is evident that the identified resonant acceleration (Cape Mendocino) produces more damage than the Landers acceleration. In fact, the three structures are damaged beyond repair $\left(D I_{\mathrm{PA}}>0.40\right)$ by the first acceleration while they sustain the second acceleration with repairable damage $\left(D I_{\mathrm{PA}}<0.40\right)$ (Park et al., 1985). The same observation applies to the ductility factor.

We further examine the measures developed in this study for the four groups of earthquake records listed in Table 5. These records include near-fault accelerograms measured at rock and soil sites, and short-duration and long-duration earthquakes (PEER 2005). The selection of records for different soil conditions and for different earthquake durations is meant for examining the robustness of the proposed measures to different earthquake characteristics. The two horizontal accelerations are considered in the numerical analyses. Table 5 provides information on these records. Note that all records are scaled to the same Arias intensity.

The numerical results on entropy and dispersion indices for each of these earthquake 
accelerations are provided in Table 5. The results reveal that the entropy and the dispersion indices correlate well and that they both successfully identify resonant accelerograms. It is seen that the narrow-band records possess the smallest entropy and dispersion indices (e.g., 1995 Kobe (OSAJ) and 1994 Northridge (Sylmar) earthquakes) while the accelerations that are rich in frequency content (e.g. 1992 Landers and 1986 N. Palm spring earthquakes) possess the largest entropy and dispersion. It is also evident that the two acceleration components of the same earthquake have different entropy and dispersion indices. This observation is consistent with the findings of Anderson and Bertero (1987). The short-duration earthquakes are seen to possess higher entropy and dispersion and sharp energy jump compared to the long-duration earthquakes. It is also remarkable that near-fault records measured on soil site have smaller entropy and dispersion indices compared to near-fault records measured on rock soil. This may be attributed to the site soil effects that can significantly filter the amplitude and frequency content of the ground motion for soil sites.

\section{CONCLUSIONS}

This paper develops measures to identify resonance of earthquake ground motions. The entropy and dispersion indices are shown to successfully identify resonance of probabilistic earthquake models and recorded accelerograms. It is shown that resonant accelerations exist regardless of soil site condition and source characteristics. It is also shown that the band-limited and the narrow-band signals provide upper and lower bounds on entropy and dispersion indices of the Kanai-Tajimi acceleration models. Similarly, resonant harmonic time functions and broad-band harmonic functions provide bounds on the frequency content of recorded accelerograms. Measures that are based on deterministic approach are also shown to be good descriptors of resonance of ground motions.

The usefulness of the measures developed in this paper is demonstrated by identifying resonant accelerations at sites with different soil conditions and earthquake characteristics. Such accelerations are shown to produce large structural damage when their dominant frequency is close to the structure fundamental frequency. Numerical verifications using 
nonlinear dynamic analysis and Park and Ang damage indices are provided.

\section{References}

Abbas, A.M. (2002) "Deterministic/reliability-based critical earthquake load models for linear/nonlinear structures," Ph D thesis, Indian Institute of Science, Bangalore.

Abbas, A.M. (2006) "Critical seismic load inputs for simple inelastic structures," Journal of Sound and Vibration 296, 949-967.

Abbas, A.M. and Manohar, C.S. (2002) "Investigations into critical earthquake excitations within deterministic and probabilistic frameworks," Earthquake Engineering \& Structural Dynamics 31, 813-832.

A.M. Abbas, (2006) Critical seismic load inputs for simple inelastic structures, Journal of Sound and Vibration, 296, 949-967.

Abbas, A.M. and Manohar, C.S. (2007) "Reliability-based vector nonstationary random critical earthquake excitations for parametrically excited systems," Structural Safety 29, $32-48$.

Amiri, G.G., and Dana, F.M. (2005) "Introduction to the most suiTable parameter for selection of critical earthquakes," Computers \& Structures 83, 613-626.

Anderson, J.C. and Bertero, V.V. (1987) "Uncertainties in establishing design earthquakes," Journal of Structural Engineering 113(8), 1709-1724.

Arias, A. (1970) "A measure of earthquake intensity," In seismic design of nuclear power plants, Cambridge, MA: MIT press, 438-468.

Dhakal R.P., mander J.B. and Mashiko N (2006) "Identification of critical ground motions for seismic performance assessment of structures” Earth. Eng. Stuct. Dyn., 35, 989-1008.

Kanai, K. (1957) "Semi-empirical formula for the seismic characteristics of the ground," Tokyo Bulletin of Earthquake Research Institute 35, 309-325.

Kapur, J.N. (1993) "Maximum entropy models in science and engineering," Wiley Eastern, New Delhi.

Lin, Y.K., (1967) "Probabilistic theory of structural dynamics," McGraw-Hill, NY.

Manohar, C.S., and Sarkar, A., (1995) "Critical earthquake input power spectral density function models for engineering structures," Earthq. Eng. Struct. Dyn. 24, 1549-1566.

Nigam, N.C. and Narayana, S. (1994) “Applications of random vibrations," Narosa Publishing House, New Delhi.

Pacific Earthquake Engineering Research Center (2005) "http://peer.berkeley.edu,".

Papoulis, A. (1991) "Probability, random variables and stochastic processes," McGraw-Hill, NY.

Park, Y.J., Ang, A H-S. (1985) "Mechanistic seismic damage model for reinforced concrete." Journal of Structural Engineering, 111(4), 722-739.

Shannon, C. (1948) "A mathematical theory of communication,” Bell Sys. Tec. J., 27.

Tajimi, H. (1960) "A statistical method of determining the maximum response of a building structure during earthquakes." Proc. 2nd WCEE, Tokyo 2, 781:797. 
Takewaki, I. (2001). "Resonance and criticality measure of ground motions via probabilistic critical excitation method.", So. Dyn. Earthq. Eng., 21, 645-659.

Takewaki, I. (2002) Seismic critical excitation method for robust design: A review, Journal of Structural Engineering 128, 665-672.

Takewaki, I., (2004). “Bound of earthquake input energy.”, J. Struct. Eng., 130(9):1289-1297.

Takewaki, I. (2007). Critical excitation methods in earthquake engineering, Elsevier Science.

Vanmarcke, E.H. (1972) "Properties of spectral moments with applications to random processes." Journal of Engineering Mechanics 42, 215-220.

Vanmarcke, E.H. (1976) "Structural response to earthquakes: Seismic risk and engineering decisions," Lomnitz, C. and Rosenbluth, E., (eds), Elsevier, NY.

Uang C-M and Bertero VV (1988) Implications of recorded earthquake ground motions on seismic design of building structures, Report No. UCB/EERC-88/13, Earthquake Engineering Research center, Berkeley, CA.

Zhai, C-H., and Xie, L-L., (2007). "A new approach of selecting real input ground motions for seismic design: The most unfavourable real seismic design ground motions.", Earth. Eng. Stuct. Dyn., 36, 1009-1027. 


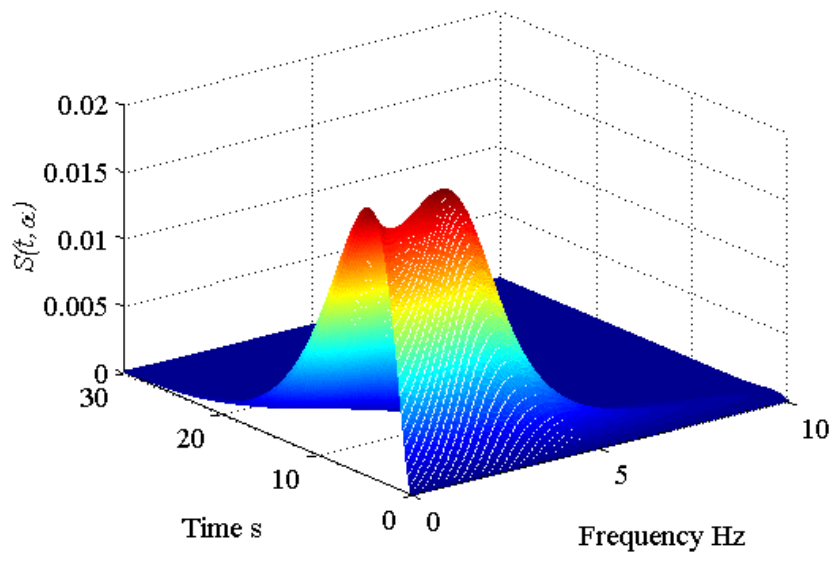

(a)

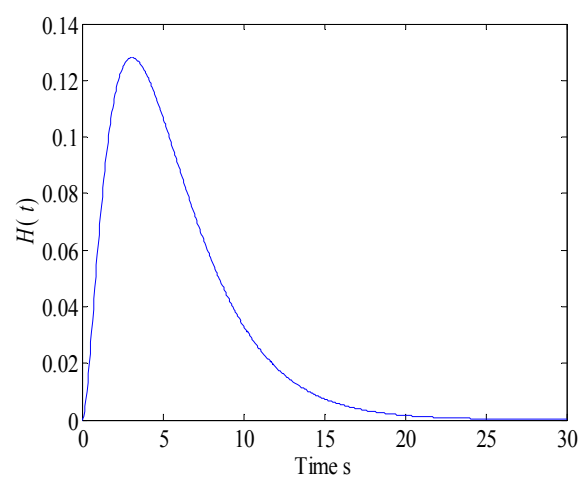

(b)

Fig. 1: (a) Evolutionary Kanai-Tajimi PSDF (b) Entropy function 
(a)

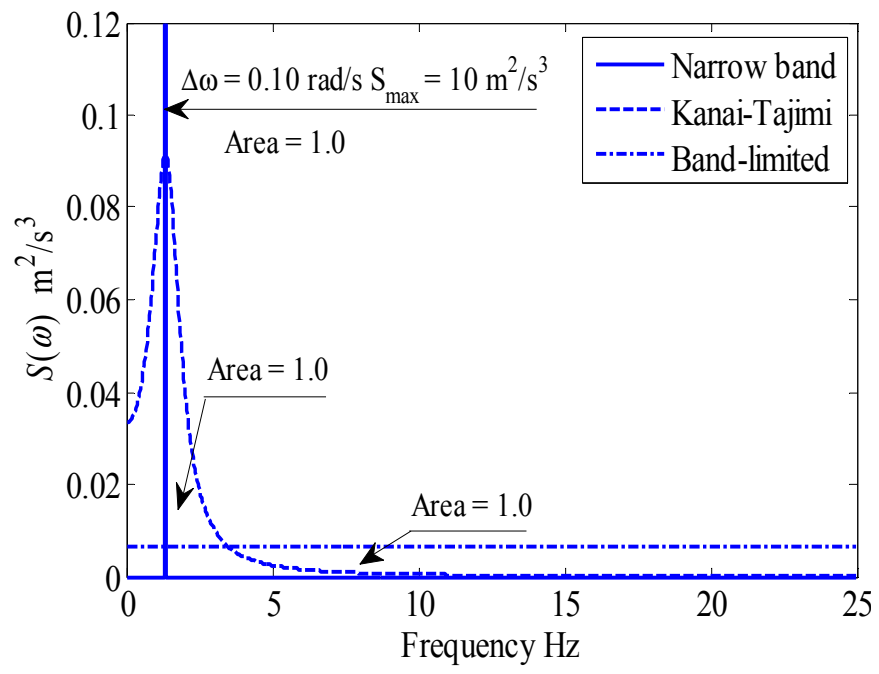

(b)

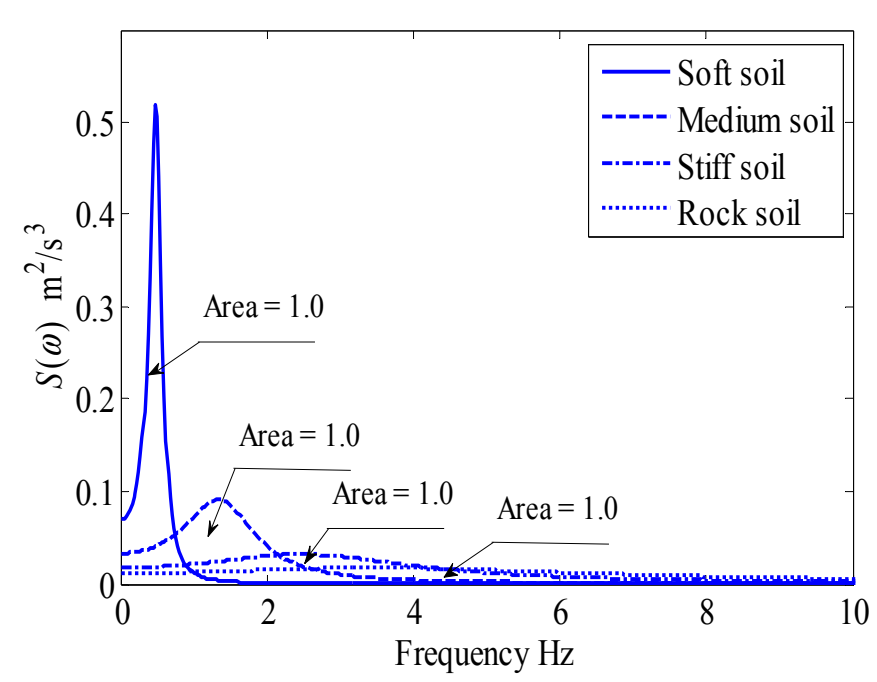

Fig. 2: (a) PSD function for ground acceleration models for medium soil (b) Kanai-Tajimi PSD function for different soil types. 
(a)

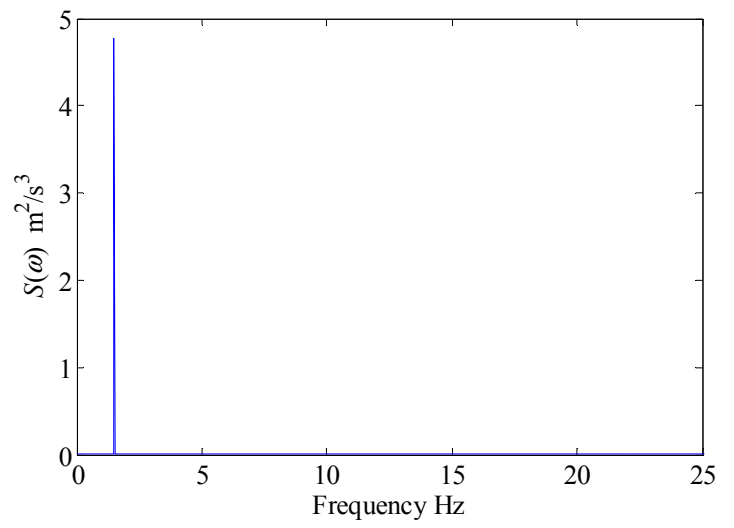

(c)

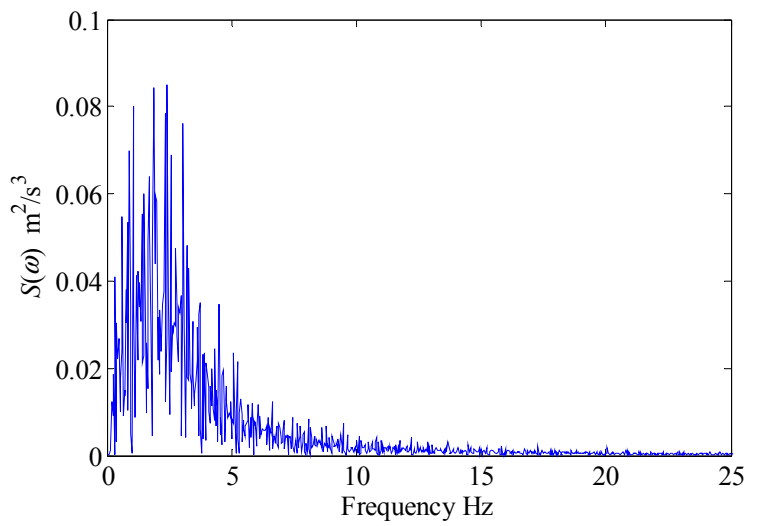

(b)

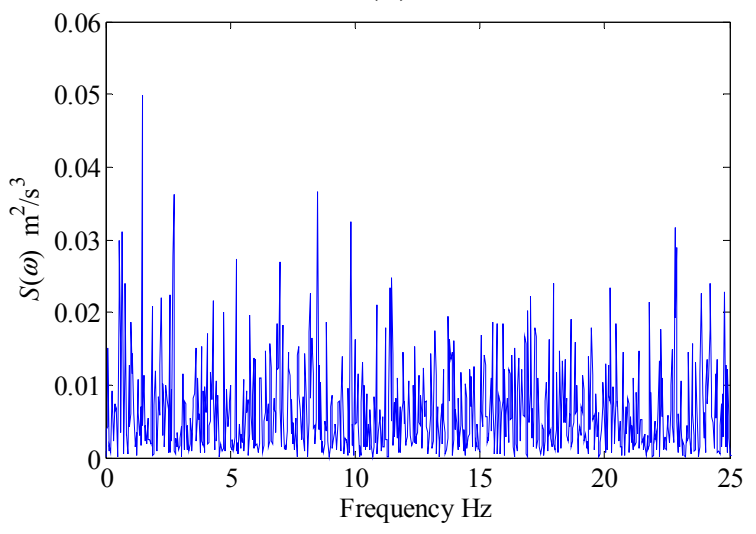

(d)

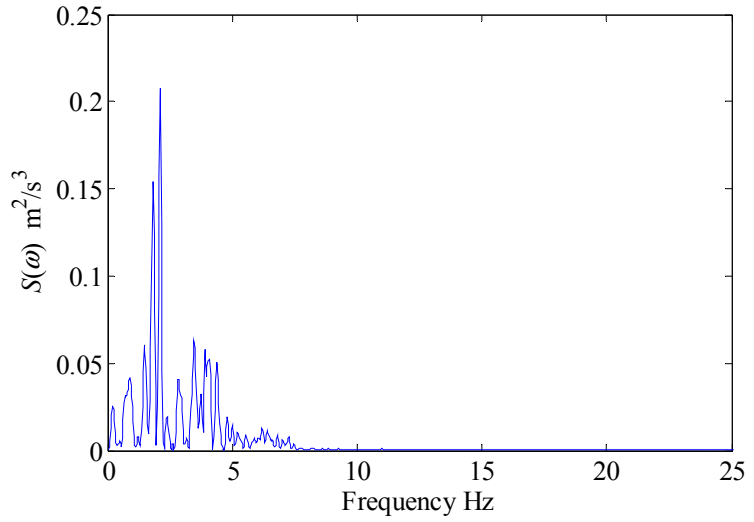

Fig. 3: PSD function for ground acceleration (a) narrow-band (b) band-limited (c) Kanai-Tajimi (d) 1992 Cape Mendocino (Petrolia) earthquake. 


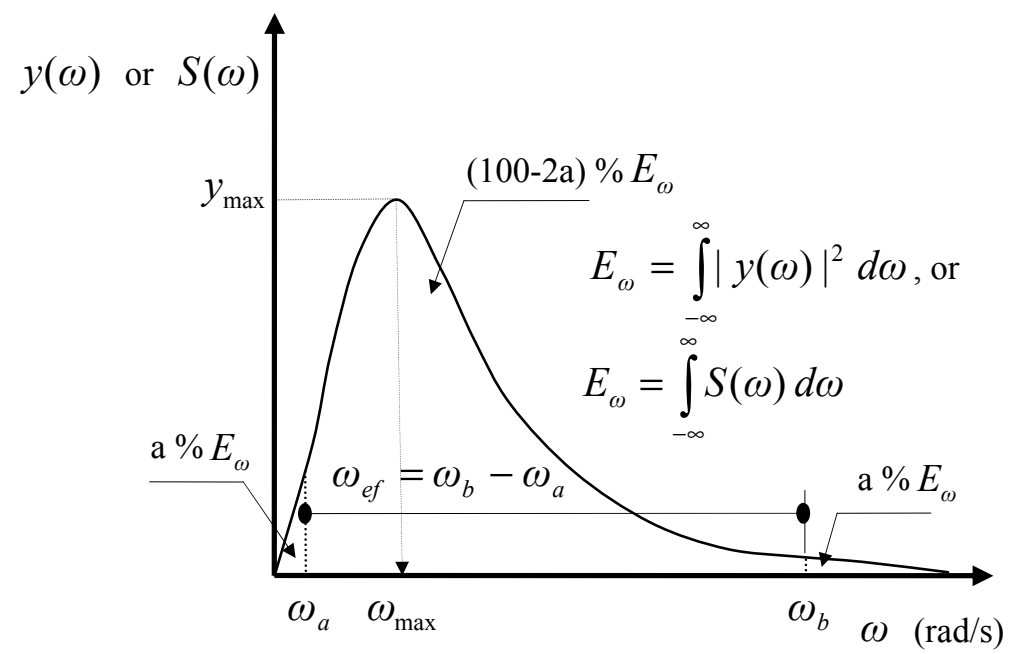

Fig. 4: Measures of frequency bandwidth of recorded ground accelerations 

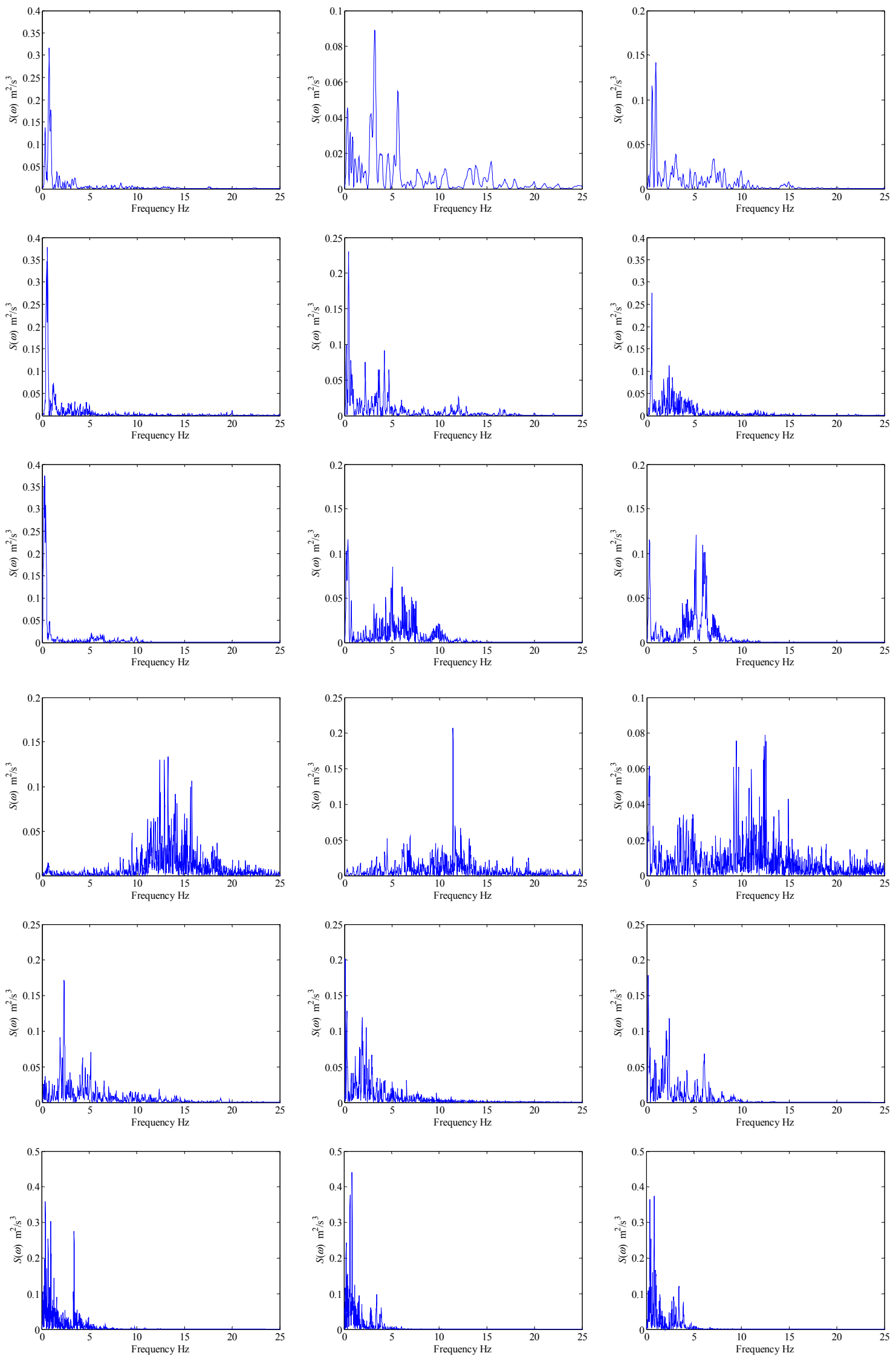

Fig. 5: Power spectral density function: records at rock soil site. 

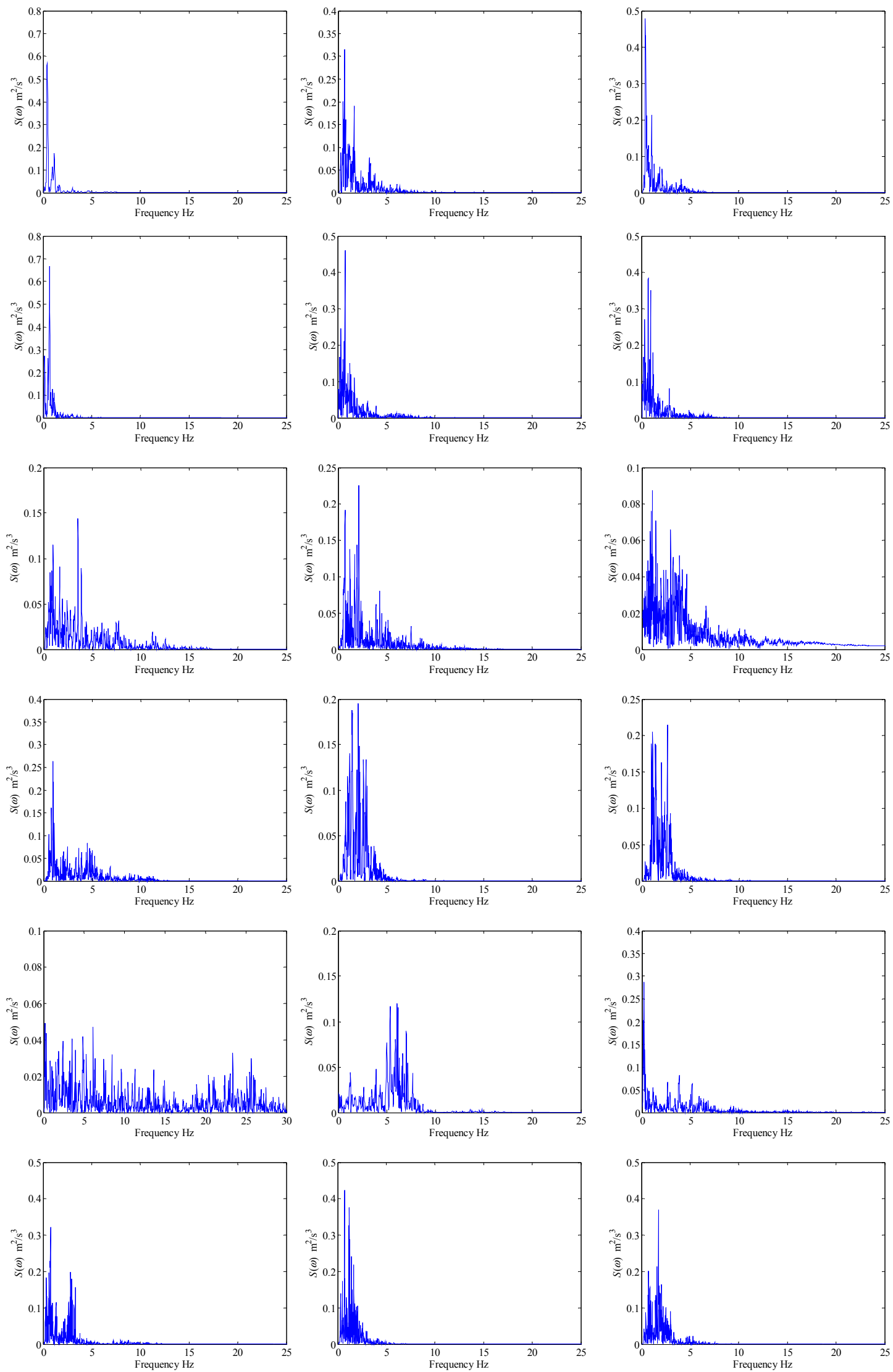

Fig. 6: Power spectral density function: records at stiff soil site. 

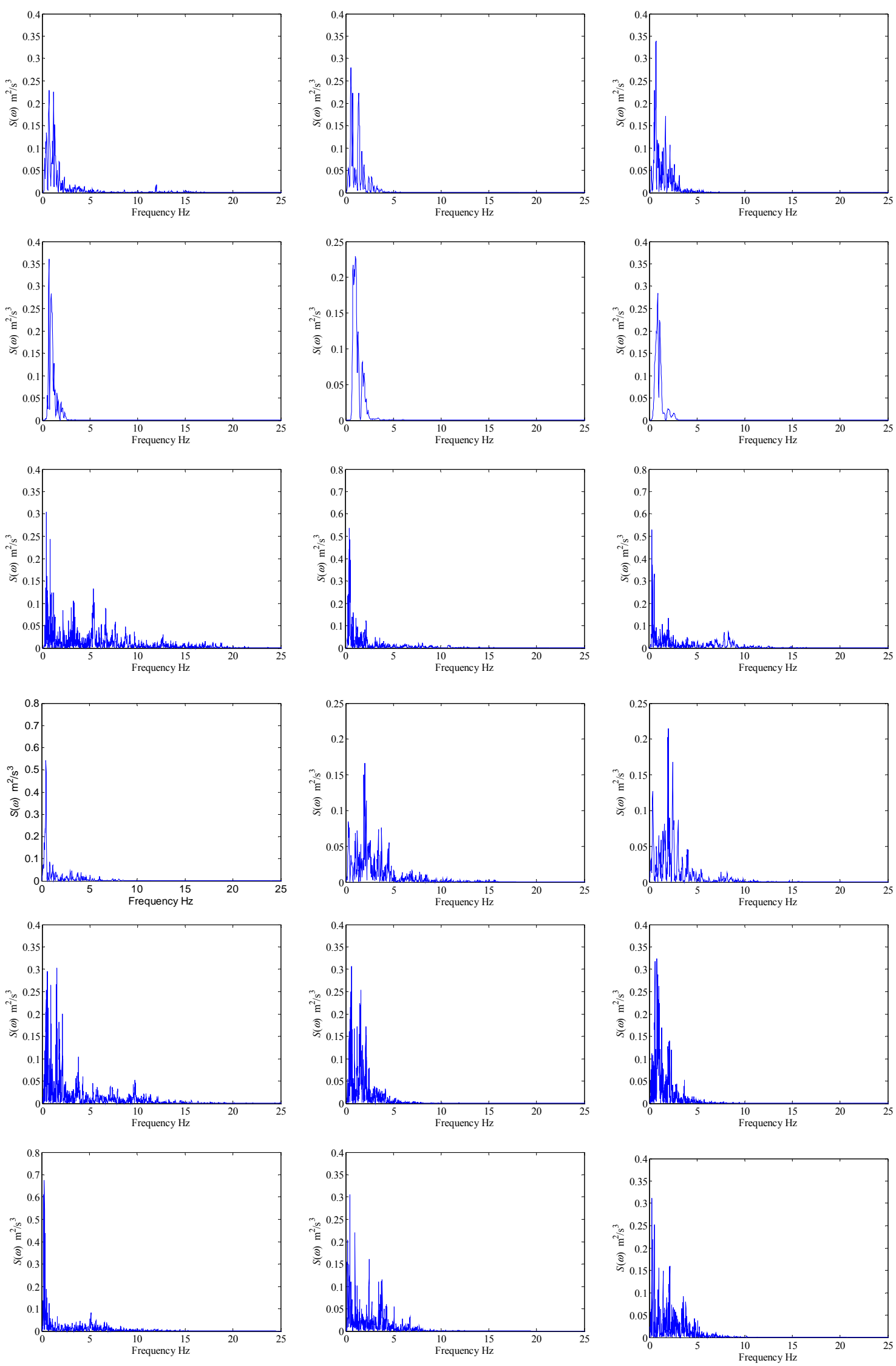

Fig. 7: Power spectral density function: records at medium soil site. 

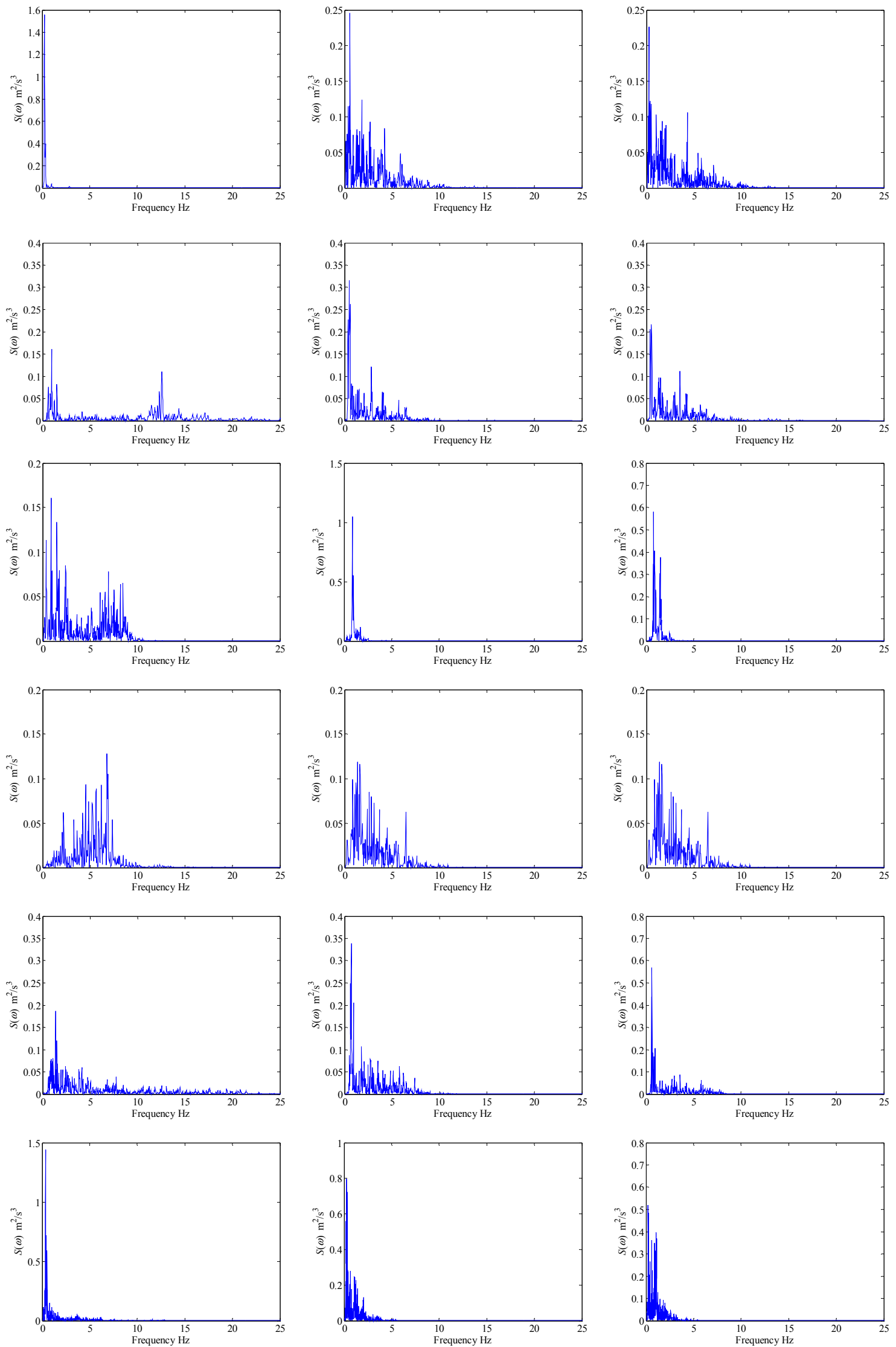

Fig. 8: Power spectral density function: records at soft soil site. 
Table 1: Resonance measures for alternative earthquake models and different soil types.

\begin{tabular}{ccccccccc}
\hline \multirow{2}{*}{ Earthquake Model } & \multicolumn{9}{c}{ Soft soil } & \multicolumn{2}{c}{ Medium soil } & \multicolumn{2}{c}{ Stiff soil } & \multicolumn{2}{c}{ Rock soil } \\
\cline { 2 - 8 } & $\begin{array}{c}\text { Entropy } \\
\text { index }\end{array}$ & $\begin{array}{c}\text { Bandwidth } \\
\text { factor }\end{array}$ & $\begin{array}{c}\text { Entropy } \\
\text { index }\end{array}$ & $\begin{array}{c}\text { Bandwidth } \\
\text { factor }\end{array}$ & $\begin{array}{c}\text { Entropy } \\
\text { index }\end{array}$ & $\begin{array}{c}\text { Bandwidth } \\
\text { factor }\end{array}$ & $\begin{array}{c}\text { Entropy } \\
\text { index }\end{array}$ & $\begin{array}{c}\text { Bandwidth } \\
\text { factor }\end{array}$ \\
\hline Narrow-band* & 0.0294 & 0.0785 & 0.0294 & 0.0785 & 0.0294 & 0.0785 & 0.0294 & 0.0785 \\
Kanai-Tajimi** & 0.2122 & 4.0869 & 0.4080 & 14.7227 & 0.5228 & 26.2513 & 0.5704 & 34.1420 \\
Band-limited & 0.6040 & 45.2997 & 0.6040 & 45.2997 & 0.6040 & 45.2997 & 0.6040 & 45.2997 \\
$\begin{array}{c}\text { 1992 Cape Mend. } \\
\text { (Petrolia) }\end{array}$ & - & - & 0.3215 & 5.7164 & - & - & - & - \\
\hline
\end{tabular}

* $\omega_{c}=\pi, 3 \pi, 6 \pi, 9 \pi$ for soft, medium, stiff and rock soil, respectively.

** $\omega_{g}=\pi, 3 \pi, 6 \pi, 9 \pi$ and $\eta_{g}=0.20,0.40,0.60,0.80$ for soft, medium, stiff and rock soil, respectively. 
Table 2: Frequency content measures for recorded ground motion.

\begin{tabular}{lcccc}
\hline \multicolumn{1}{c}{ Earthquake (station, record) } & $\omega_{c}(\mathrm{~Hz})$ & $\omega_{\text {ef }}(\mathrm{Hz})$ & $\bar{\omega}_{e f} *$ & $\omega_{\max }(\mathrm{Hz})$ \\
\hline 1940 Elcentro (El Centro\#9, H180) & 13.53 & 43.92 & 0.88 & 5.88 \\
1966 Parkfield (Cholame\#2, C065) & 8.18 & 28.85 & 0.58 & 8.24 \\
1992 Landers (Lucerne, LCN000) & 23.05 & 46.69 & 0.93 & 28.52 \\
1995 Kobe (Takatori, TAK000) & 6.75 & 31.49 & 0.63 & 4.03 \\
1999 Chichi (ALS, ALS0) & 3.83 & 16.52 & 0.33 & 1.74 \\
\hline
\end{tabular}

*Equation (4), $\Omega_{0}=0, \Omega_{u}=50 \mathrm{~Hz}$. 
Table 3: Information on earthquake records and entropy and bandwidth indices for different soil conditions [32].

\begin{tabular}{|c|c|c|c|c|c|c|c|c|c|}
\hline Soil type & Farthauake (station) & $M^{*}$ & Ep. dis. & PGA $(g)$ & $I_{A}^{* *}\left(\mathrm{~m}^{2} / \mathrm{s}^{3}\right)$ & Dur. & Ef. Bandwidth & Entropy $^{* * *}$ & Band. Factor $^{* * * *}$ \\
\hline$\left(v_{s}\right.$ & Eartnquake (station) & & $(\mathrm{km})$ & $\mathrm{V} / \mathrm{H}_{1} / \mathrm{H}_{2}$ & $\mathrm{~V} / \mathrm{H}_{1} / \mathrm{H}_{2}$ & (s) & $\mathrm{V} / \mathrm{H}_{1} / \mathrm{H}_{2}$ & $\mathrm{~V} / \mathrm{H}_{1} / \mathrm{H}_{2}$ & $\mathrm{~V} / \mathrm{H}_{1} / \mathrm{H}_{2}$ \\
\hline \multirow{6}{*}{$\begin{array}{c}\text { Rock } \\
v_{s}>750\end{array}$} & 1971 San Fernando (Lake Hug. \#9) & 6.6 & 23.10 & $0.09 / 0.16 / 0.13$ & $0.50 / 0.94 / 0.67$ & 34.89 & $0.63 / 0.57 / 0.54$ & $0.49 / 0.70 / 0.66$ & $1.46 / 0.83 / 0.90$ \\
\hline & 1989 Loma Prieta (Gilory \#1) & 6.9 & 28.64 & $0.21 / 0.41 / 0.47$ & $1.85 / 6.59 / 10.49$ & 39.95 & $0.64 / 0.34 / 0.42$ & $0.47 / 0.62 / 0.60$ & $1.50 / 1.03 / 0.98$ \\
\hline & 1992 Cape Mendocino (CM) & 7.1 & 10.36 & $0.75 / 1.50 / 1.04$ & $8.59 / 37.19 / 14.91$ & 30.00 & $0.44 / 0.34 / 0.39$ & $0.42 / 0.63 / 0.58$ & $1.64 / 1.03 / 0.98$ \\
\hline & 1992 Landers (Lurcene) & 7.3 & 44.02 & $0.82 / 0.72 / 0.78$ & $51.37 / 41.10 / 43.46$ & 48.13 & $0.79 / 0.77 / 0.81$ & $0.62 / 0.68 / 0.71$ & $0.29 / 0.41 / 0.56$ \\
\hline & 1999 Kocaeli (ERD) & 7.4 & 47.03 & $0.20 / 0.24 / 0.14$ & $1.16 / 3.43 / 2.00$ & 28.00 & $0.48 / 0.23 / 0.24$ & $0.67 / 0.63 / 0.58$ & $0.84 / 1.18 / 0.89$ \\
\hline & 1999 Chichi (TAP051) & 7.6 & 152.71 & $0.04 / 0.06 / 0.11$ & $0.27 / 0.81 / 1.44$ & 90.00 & $0.22 / 0.17 / 0.17$ & $0.46 / 0.43 / 0.49$ & $0.84 / 0.90 / 0.81$ \\
\hline \multirow{6}{*}{$\begin{array}{c}\text { Stiff soil } \\
360<v_{s} \leq 750\end{array}$} & 1989 Loma Prieta (Gilory \#6) & 6.9 & 35.47 & $0.10 / 0.13 / 0.17$ & $0.61 / 1.39 / 2.76$ & 39.96 & $0.38 / 0.29 / 0.19$ & $0.37 / 0.50 / 0.43$ & $1.58 / 1.09 / 1.21$ \\
\hline & 1992 Cape Mendocino (Fort. Blvd) & 7.1 & 29.55 & $0.05 / 0.12 / 0.11$ & $0.34 / 1.63 / 1.49$ & 44.00 & $0.33 / 0.17 / 0.16$ & $0.34 / 0.48 / 0.45$ & $1.53 / 1.24 / 1.14$ \\
\hline & 1992 Landers (DH Springs) & 7.3 & 27.33 & $0.17 / 0.17 / 0.15$ & $3.61 / 4.41 / 4.23$ & 50.00 & $0.40 / 0.26 / 0.24$ & $0.64 / 0.57 / 0.71$ & $0.82 / 0.88 / 0.81$ \\
\hline & 1995 Kobe (0KJMA) & 6.9 & 18.27 & $0.34 / 0.82 / 0.60$ & $11.66 / 52.40 / 33.94$ & 48.00 & $0.22 / 0.12 / 0.14$ & $0.60 / 0.46 / 0.46$ & $0.76 / 0.57 / 0.63$ \\
\hline & 1999 Kocaeli (Arcelik) & 7.4 & 53.68 & $0.09 / 0.22 / 0.15$ & $0.50 / 1.81 / 1.36$ & 30.00 & $0.65 / 0.34 / 0.37$ & $0.77 / 0.61 / 0.63$ & $0.79 / 0.52 / 1.10$ \\
\hline & 1999 Chichi (ALS) & 7.6 & 37.83 & $0.07 / 0.18 / 0.16$ & $1.26 / 6.00 / 5.74$ & 59.00 & $0.24 / 0.14 / 0.15$ & $0.48 / 0.42 / 0.47$ & $0.94 / 0.78 / 0.69$ \\
\hline \multirow{6}{*}{$\begin{array}{l}\text { Medium soil } \\
180 \leq v_{s} \leq 360\end{array}$} & 1942 Borrego (Elcentro \#9) & 6.5 & 57.79 & $0.04 / 0.07 / 0.10$ & $0.08 / 0.53 / 0.39$ & 40.00 & $0.46 / 0.24 / 0.23$ & $0.48 / 0.40 / 0.43$ & $1.46 / 0.74 / 0.85$ \\
\hline & 1960 Central Calif. (Hollister CH) & 5.0 & 8.01 & $0.03 / 0.04 / 0.06$ & $0.06 / 0.12 / 0.26$ & 40.00 & $0.17 / 0.13 / 0.15$ & $0.33 / 0.35 / 0.45$ & $0.49 / 0.57 / 0.55$ \\
\hline & 1992 Big Bear (SBE \& H) & 6.4 & 45.51 & $0.07 / 0.09 / 0.10$ & $0.65 / 1.70 / 1.83$ & 100.00 & $0.38 / 0.23 / 0.26$ & $0.54 / 0.45 / 0.53$ & $0.69 / 1.03 / 0.88$ \\
\hline & 1992 Cape Mendocino (Petrolia) & 7.1 & 4.51 & $0.16 / 0.59 / 0.66$ & $2.46 / 21.33 / 23.86$ & 36.00 & $0.32 / 0.33 / 0.33$ & $0.46 / 0.58 / 0.53$ & $1.42 / 0.89 / 0.90$ \\
\hline & 1999 Chichi (CHY006) & 7.6 & 40.47 & $0.20 / 0.36 / 0.35$ & $3.98 / 12.70 / 9.35$ & 150.00 & $0.45 / 0.21 / 0.17$ & $0.45 / 0.45 / 0.44$ & $0.71 / 0.64 / 0.66$ \\
\hline & 1999 Chichi (CHY024) & 7.6 & 24.10 & $0.15 / 0.18 / 0.28$ & $11.41 / 7.46 / 4.33$ & 90.00 & $0.29 / 0.22 / 0.19$ & $0.51 / 0.53 / 0.49$ & $1.03 / 0.81 / 0.73$ \\
\hline \multirow{6}{*}{$\begin{array}{c}\text { Soft soil } \\
v_{s}<180\end{array}$} & 1979 Imperial Valley (Elcentro \#3) & 6.5 & 28.65 & $0.13 / 0.27 / 0.22$ & $1.32 / 7.08 / 4.26$ & 39.55 & $0.48 / 0.26 / 0.25$ & $0.20 / 0.59 / 0.58$ & $2.50 / 0.85 / 0.92$ \\
\hline & 1981 Westmorland (SSW Rd.) & 5.8 & 8.62 & $0.21 / 0.20 / 0.18$ & $2.05 / 3.26 / 3.20$ & 28.75 & $0.93 / 0.33 / 0.35 /$ & $0.70 / 0.51 / 0.57$ & $0.67 / 1.04 / 0.99$ \\
\hline & 1989 Loma Prieta (Apeel2 RC) & 6.9 & 63.49 & $0.08 / 0.27 / 0.22$ & $0.58 / 7.82 / 4.44$ & 35.83 & $0.20 / 0.10 / 0.10$ & $0.61 / 0.32 / 0.31$ & $0.71 / 1.04 / 0.57$ \\
\hline & 1994 Northridge (MB Rd.) & 6.7 & 47.11 & $0.08 / 0.18 / 0.13$ & $0.41 / 2.02 / 1.20$ & 21.83 & $0.26 / 0.19 / 0.21$ & $0.59 / 0.54 / 0.58$ & $0.46 / 0.56 / 0.77$ \\
\hline & 1995 Kobe (Kakogawa) & 6.9 & 24.20 & $0.16 / 0.25 / 0.35$ & $1.75 / 6.44 / 10.54$ & 40.96 & $0.44 / 0.23 / 0.22$ & $0.68 / 0.54 / 0.51$ & $0.84 / 0.82 / 0.88$ \\
\hline & 1999 Kocaeli (Ambarli) & 7.4 & 112.26 & $0.08 / 0.25 / 0.18$ & $0.86 / 6.28 / 7.74$ & 150.41 & $0.40 / 0.10 / 0.10$ & $0.35 / 0.37 / 0.37$ & $1.11 / 0.90 / 0.76$ \\
\hline
\end{tabular}

${ }^{*} M=$ Richter's magnitude.

${ }^{* *} I_{A}=\int_{0}^{\infty}\left[\ddot{x}_{g}(t)\right]^{2} d t$ (Arias intensity).

*** Entropy index calculated from Eq. (5).

**** Bandwidth factor calculated from Eq. (9). 
Table 4: Ductility factor and damage index for SDOF inelastic structure subjected to ground acceleration.

\begin{tabular}{lccccccc}
\hline \multirow{2}{*}{ Earthquake (station) } & \multicolumn{6}{c}{ Structure fundamental frequency } \\
\cline { 2 - 7 } & \multicolumn{2}{c}{$f=0.13 \mathrm{~Hz}$} & \multicolumn{2}{c}{$f=0.21 \mathrm{~Hz}$} & \multicolumn{2}{c}{$f=0.41 \mathrm{~Hz}$} \\
\cline { 2 - 7 } & $\begin{array}{c}\text { Ductility } \\
\text { factor }\end{array}$ & $\begin{array}{c}\text { Damage } \\
\text { index }\end{array}$ & $\begin{array}{c}\text { Ductility } \\
\text { factor }\end{array}$ & $\begin{array}{c}\text { Damage } \\
\text { index }\end{array}$ & $\begin{array}{c}\text { Ductility } \\
\text { factor }\end{array}$ & $\begin{array}{c}\text { Damage } \\
\text { index }\end{array}$ \\
\hline 1992 Cape Mendocino (CM) & 4.39 & 0.74 & 7.09 & 1.05 & 1.41 & 0.19 \\
1992 Landers (Lurcene) & 0.93 & 0.12 & 0.60 & 0.08 & 0.43 & 0.05 \\
\hline
\end{tabular}


Table 5: Information on earthquake records and entropy and dispersion indices for different soil conditions (PEER 2005).

\begin{tabular}{|c|c|c|c|c|c|c|c|c|}
\hline Group type & Earthquake (station) & $M_{W}$ & PGA (g) & $I_{A}{ }^{*}\left(\mathrm{~m}^{2} / \mathrm{s}^{3}\right)$ & Duration (s) & $\begin{array}{c}\text { Ef. Bandwidth } \\
\mathrm{H}_{1} / \mathrm{H}_{2}\end{array}$ & $\begin{array}{c}\text { Entropy index } \\
\mathrm{H}_{1}{ }^{* *} / \mathrm{H}_{2}\end{array}$ & $\begin{array}{c}\text { Dispersion index } \\
\mathrm{H}_{1} \quad / \mathrm{H}_{2} \\
\end{array}$ \\
\hline \multirow{4}{*}{$\begin{array}{l}\text { Near-fault } \\
\text { (rock) }\end{array}$} & 1992 Landers (Lucerne) & 7.2 & $0.79 / 0.72$ & $41.10 / 43.46$ & 48.13 & $0.77 / 0.81$ & $0.68 / 0.71$ & $27.90 / 34.85$ \\
\hline & 1992 Cape Mendocino (CM) & 7.0 & $1.50 / 1.04$ & $37.19 / 14.91$ & 30.00 & $0.39 / 0.34$ & $0.63 / 0.58$ & $21.34 / 15.93$ \\
\hline & 1995 Kobe (JMA) & 6.9 & $0.82 / 0.60$ & $52.40 / 33.94$ & 48.00 & $0.13 / 0.14$ & $0.46 / 0.46$ & $7.73 / 8.30$ \\
\hline & 1989 Loma Prieta (LG pres. center) & 6.9 & $0.61 / 0.56$ & $49.12 / 19.28$ & 24.97 & $0.29 / 0.34$ & $0.48 / 0.56$ & $17.79 / 22.29$ \\
\hline \multirow{5}{*}{$\begin{array}{l}\text { Near-fault } \\
\text { (soil) }\end{array}$} & 1978 Tabas (Tabas) & 7.4 & $0.84 / 0.85$ & $46.03 / 24.74$ & 32.84 & $0.28 / 0.26$ & $0.67 / 0.60$ & $27.64 / 17.30$ \\
\hline & 1992 Cape Mendocino (Petrolia) & 7.0 & $0.59 / 0.66$ & $21.33 / 23.86$ & 36.00 & $0.33 / 0.32$ & $0.58 / 0.53$ & $17.51 / 14.82$ \\
\hline & 1994 Northridge (Rinaldi) & 6.7 & $0.84 / 0.85$ & $72.09 / 72.01$ & 14.95 & $0.23 / 0.35$ & $0.59 / 0.64$ & $23.01 / 28.15$ \\
\hline & 1994 Northridge (Sylmar converter) & 6.7 & $0.61 / 0.90$ & $36.42 / 32.99$ & 40.00 & $0.22 / 0.19$ & $0.33 / 0.37$ & $5.27 / 9.23$ \\
\hline & 1979 Imperial valley (Meloland) & 6.5 & $0.31 / 0.30$ & $5.36 / 6.74$ & 39.99 & $0.17 / 0.13$ & $0.48 / 0.41$ & $9.88 / 9.71$ \\
\hline \multirow{6}{*}{ Short-duration } & 1983 Caolinga (Anticline Ridge) & 5.0 & $0.67 / 0.58$ & $4.42 / 3.90$ & 9.84 & $0.36 / 0.42$ & $0.55 / 0.54$ & $18.09 / 24.87$ \\
\hline & 1970 Lytle Creek (Devils canyon) & 5.4 & $0.15 / 0.15 /$ & $0.65 / 0.57$ & 13.61 & $0.34 / 0.43$ & $0.40 / 0.46$ & $20.33 / 24.56$ \\
\hline & 1986 N. Palm Spring (SR Mountain) & 6.0 & $0.10 / 0.10$ & $0.29 / 0.39$ & 10.40 & $0.97 / 0.99$ & $0.79 / 0.82$ & $70.27 / 70.42$ \\
\hline & 1975 Northern Calif. (CM Petrolia) & 5.2 & $0.18 / 0.12$ & $0.74 / 0.55$ & 14.61 & $0.28 / 0.22$ & $0.58 / 0.53$ & $15.26 / 12.53$ \\
\hline & 1971 San Fernando (Gormon-Oso) & 6.6 & $0.11 / 0.08$ & $0.37 / 0.28$ & 9.23 & $0.21 / 0.23$ & $0.64 / 0.70$ & $17.68 / 13.76$ \\
\hline & 1984 Morgan Hill (Lick observatory) & 6.2 & $0.05 / 0.08$ & $0.15 / 0.50$ & 16.00 & $0.20 / 0.18$ & $0.58 / 0.53$ & $15.93 / 17.05$ \\
\hline \multirow{4}{*}{ Long-duration } & 1992 Big Bear (SB-E \& hospitality) & 6.4 & $0.10 / 0.10$ & $1.70 / 1.83$ & 100.00 & $0.23 / 0.26$ & $0.45 / 0.53$ & $11.26 / 14.12$ \\
\hline & 1995 Kobe (OSAJ) & 6.9 & $0.08 / 0.06$ & $1.44 / 1.22$ & 120.00 & $0.14 / 0.16$ & $0.19 / 0.17$ & $1.89 / 1.40$ \\
\hline & 1999 Kocaeli (Maslak) & 7.4 & $0.25 / 0.19$ & $6.28 / 7.74$ & 150.41 & $0.10 / 0.10$ & $0.37 / 0.37$ & $5.68 / 5.10$ \\
\hline & 1999 Chichi (CHY034) & 7.6 & $0.31 / 0.25$ & $11.35 / 9.12$ & 250.00 & $0.12 / 0.13$ & $0.38 / 0.35$ & $4.38 / 4.16$ \\
\hline
\end{tabular}

${ }^{*} I_{A}=\int_{0}^{\infty}\left[\ddot{x}_{g}(t)\right]^{2} d t$ (Arias intensity).

${ }_{* * *}^{* *}$ Entropy index calculated from Eq. (8).

Dispersion index calculated from Eq. (12) 